\title{
Diverse Proton-Conducting Nanotubes via a Tandem Macrocyclization and Assembly Strategy
}

Michael J. Strauss, ${ }^{1}$ Manping Jia, ${ }^{2}$ Austin M. Evans, ${ }^{1}$ Ioannina Castano, ${ }^{1}$ Rebecca L. Li, ${ }^{1}$ Xavier Aguilar-Enriquez, ${ }^{1}$ Emily K. Roesner, ${ }^{1}$ Jeremy L. Swartz, ${ }^{1}$ Anton D. Chavez, ${ }^{1,3}$ Alan E. Enciso, ${ }^{1}$ J. Fraser Stoddart, ${ }^{1,4,5,6}$ Marco Rolandi, ${ }^{2 *}$ and William R. Dichtel ${ }^{1 *}$

${ }^{1}$ Department of Chemistry, Northwestern University, 2145 Sheridan Road, Evanston, IL, 60208, USA

${ }^{2}$ Department of Electrical and Computer Engineering, Baskin School of Engineering, University of California Santa Cruz, Santa Cruz, CA, 95064, USA

${ }^{3}$ Department of Chemistry and Chemical Biology, Cornell University, Ithaca, New York 14853, USA

${ }^{4}$ School of Chemistry, University of New South Wales, Sydney, NSW 2052, Australia

${ }^{5}$ Stoddart Institute of Molecular Science, Department of Chemistry, Zhejiang University, Hangzhou 310027, China

${ }^{6}$ ZJU-Hangzhou Global Scientific and Technological Innovation Center, Hangzhou 311215, China

\begin{abstract}
Macrocycles that assemble into nanotubes exhibit emergent properties stemming from their low dimensionality, structural regularity, and distinct interior environments. Here, we report a versatile strategy to synthesize diverse nanotube structures in a single, efficient reaction by using a conserved building block bearing a pyridine ring. Imine condensation of a 2,4,6-triphenylpyridine-based diamine with various aromatic dialdehydes yields chemically distinct pentagonal [5+5], hexagonal [3+3], and diamond-shaped [2+2] macrocycles depending on the substitution pattern of the aromatic dialdehyde monomer. Atomic force microscopy and in solvo X-ray diffraction demonstrate that protonation of the macrocycles under the mild conditions used for their synthesis drives assembly into high-aspect ratio nanotubes. Each of the pyridine-containing nanotube assemblies exhibited measurable proton conductivity by electrochemical impedance spectroscopy, with values as high as $10^{-3} \mathrm{~S} \mathrm{~m}^{-1}(90 \%$ R.H., 25 ${ }^{\circ} \mathrm{C}$ ) that we attribute to differences in their internal pore sizes. This synthetic strategy represents a general method to access robust nanotube assemblies from a universal pyridine-containing monomer, which will enable systematic investigations of their emergent properties.
\end{abstract}

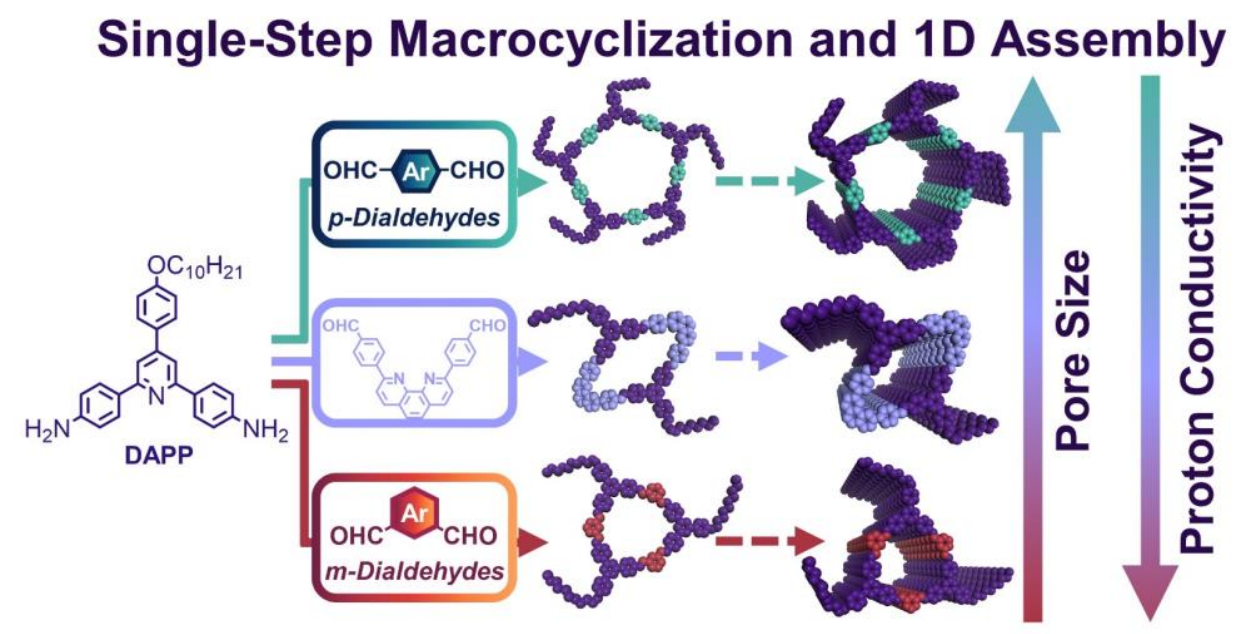




\section{Introduction}

Macrocycles that assemble into extended one-dimensional nanotubes exhibit emergent properties because of their low dimensionality, structural regularity, and distinct interior environments. ${ }^{1-6}$ These features are of potential interest for ion transport, ${ }^{7-9}$ sensing, ${ }^{10-12}$ catalysis, ${ }^{13-14}$ and separations. ${ }^{15-16}$ To access the diverse properties associated with these potential uses, macrocycles that form nanotubes must be derived from readily accessible building blocks and tolerate structural variation without compromising their interaction energies and ability to assemble. General methods to access designed, high-aspect ratio nanotubes remain limited. Non-covalent assemblies based on relatively weak supramolecular interactions are less likely to tolerate significant structural variation and can exhibit poor mechanical integrity. ${ }^{6,}$ 17-22 Developing a robust and chemically general macrocycle assembly strategy will enable broad explorations into nanotube design, their emergent properties, and stimuli-responsive ${ }^{23-26}$ or even out-of-equilibrium assembly processes. ${ }^{27-30}$

We recently found that the protonation of imine-linked macrocycles triggers strong electrostatic and solvophobic interactions that drive the formation of high-aspect ratio nanotubes. ${ }^{31-33}$ However, macrocycle assembly that relied exclusively on imine protonation only assembled in the presence of excess acid, whereas lower acid concentrations accelerated macrocycle hydrolysis. ${ }^{31-32} \mathrm{We}$ subsequently identified a single macrocycle based on pyridine-2,6-dicarboxaldehyde that assembled using sub-stoichiometric quantities of acid, giving rise to robust assemblies that formed fibers with mechanical properties comparable to covalent linear polymers (Figure 1). ${ }^{32-33}$ Here, we dramatically expand the modularity of this design by including the pyridine heterocycle and hydrophobic solubilizing groups in a single monomer, thereby enabling the efficient incorporation of many dialdehydes into macrocycles that assemble into high-aspect ratio nanotubes under mild conditions (Figure 1). Through this approach, ten distinct macrocycles and nanotubes with a range of structural features including different shapes, channel sizes, and chemical functionalities were realized. Because each of these macrocycles forms nanotubes in the presence of less than one equivalent of acid per pyridine moiety, we hypothesized that protons might be mobile within the nanotube interiors. Electrochemical impedance spectroscopy of four nanotube structures indicated that each system exhibited measurable protonic conductivity, with the highest value of $1.598 \times 10^{-3} \mathrm{~S} \mathrm{~m}^{-1}$ at $90 \%$ relative humidity and $25^{\circ} \mathrm{C}$. Across the four nanotubes studied, the protonic conductivities appear to depend on the size and/or shape of the macrocycle. These findings highlight the potential of well-defined supramolecular assemblies with precisely installed functional groups for ion transport. Overall, this versatile synthetic platform to target designed nanotubes will enable the broad 
exploration of synthetic 1D nanostructures, their emergent properties, and their eventual incorporation into devices.
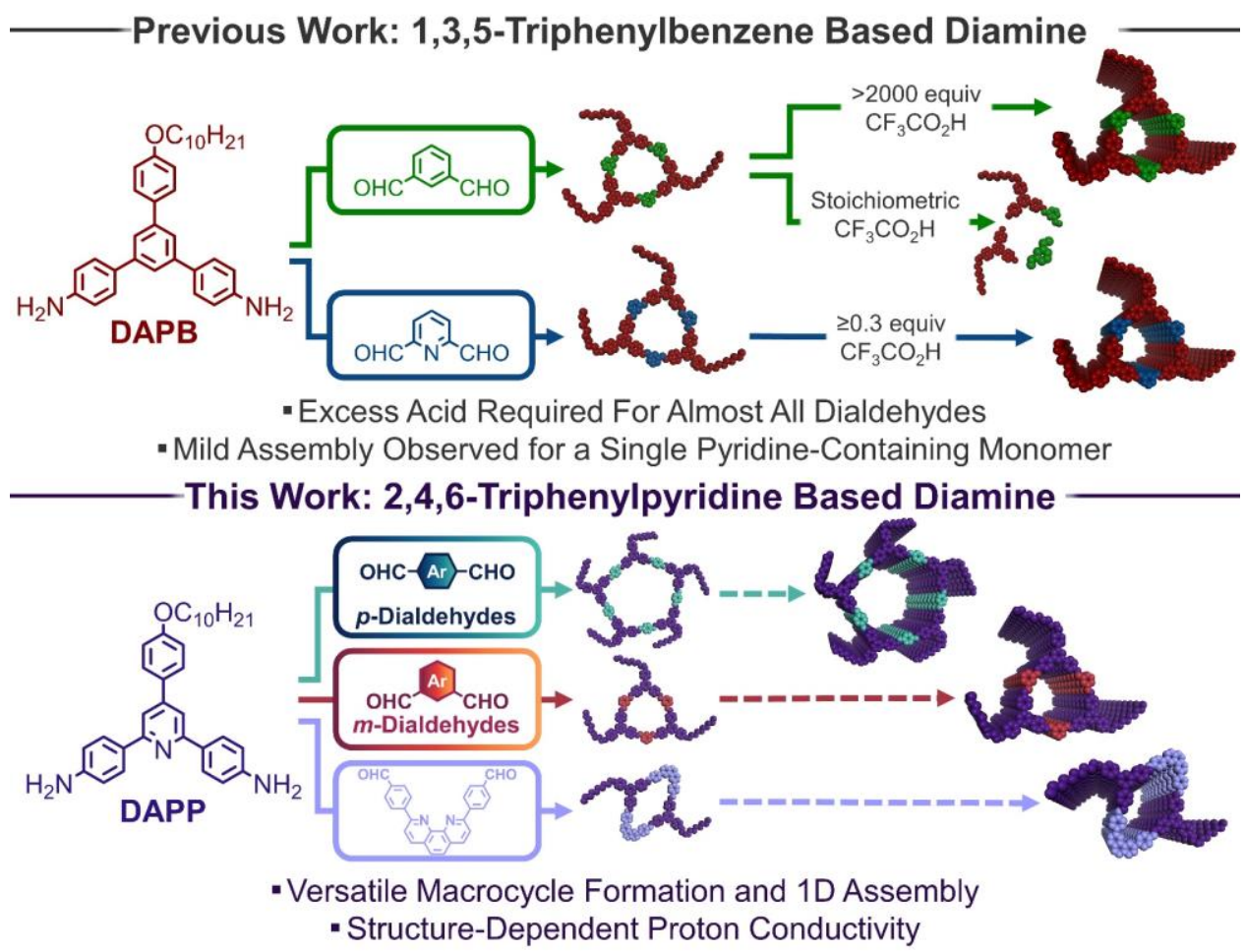

Figure 1. Accessing high-aspect ratio nanotubes via acid-mediated macrocycle assembly. (Top) Previous work in which assembly under mild conditions was dictated by a pyridine-2,6-dicarboxaldehyde monomer residue, resulting in a system that assembles under mild conditions but is not easily generalized. (Bottom) A modular approach to imine-linked macrocycle formation and assembly relying on a pyridine junction embedded within the diamine monomer, allowing access to chemically and structurally diverse nanotubes in a single step.

\section{Results and Discussion}

We prepared ten structurally diverse imine-linked macrocycles in high isolated yields $(>90 \%)$ by condensing various aromatic dialdehydes with a 2,4,6-triphenylpyridine-based diamine (DAPP) under mildly acidic conditions (Figure 2A). meta-Substituted dialdehydes yielded hexagonal macrocycles containing three diamine monomer residues and three dialdehyde monomer residues (i.e., [3+3] systems). Using this approach, macrocycles based on isophthalaldehyde (IDA) and pyridine-2,6-dicarboxaldehyde (DFP) were synthesized, similar to those in previous reports. ${ }^{33}$ This modular approach allowed access to macrocycles with externally facing functionality, 5-bromoisophthalaldehyde (5-Br-IDA) and 5ethynylisophthalaldehyde (5-E-IDA), and internal functionality, 2-bromoisophthalaldehyde (2-Br-IDA, Schemes S14-18). These groups each offer a convenient chemical handle for post-synthetic transformations, further contributing to the structural diversity available to these systems. ${ }^{34-38}$ Unlike previous reports, in which linear dialdehydes yielded [6+6] macrocycles when condensed with a 1,3,5- 
triphenylbenzene-based diamine, ${ }^{31,39}$ the condensation of linear dialdehydes with DAPP yielded pentagonal [5+5] systems. We attribute this change in macrocycle structure to the shorter $\mathrm{C}-\mathrm{N}$ bonds that make up the pyridine core of DAPP, relative to the $\mathrm{C}-\mathrm{C}$ bonds within the benzene ring of the previously studied system. ${ }^{40}$ The shorter $\mathrm{C}-\mathrm{N}$ bonds result in a decreased bite angle between the two reactive amines from $120^{\circ}$ to $108^{\circ}$, leading to the realization of a pentagonal structure. This approach yielded structurally and electronically diverse macrocycles using terephthalaldehyde (PDA), 2,3,5,6tetrafluoroterephthalaldehyde (F4-PDA), ${ }^{41-42}$ 2,5-dimethoxyterephthalaldheyde (DiOMe-PDA), ${ }^{43-44}$ and 9,10-anthracenedicarboxaldehyde (9,10-ADA) monomers (Schemes S19-22). ${ }^{45-46}$ Lastly, we prepared a diamond shaped [2+2] macrocycle from DAPP and 4,4'-(1,10-phenanthroline-2,9-diyl)dibenzaldehyde (PhenDA) which can chelate metals within the phenanthroline binding pocket (Scheme S23) ${ }^{47-48}$ The ability to target macrocycles of different sizes, chemical functionalities, and intrinsic properties highlights the modularity of this approach, and its overall utility in constructing precisely functionalized nanostructures.

Characterization of the monomeric macrocycles by gel permeation chromatography (GPC), matrixassisted laser desorption ionization mass spectrometry (MALDI-MS), and ${ }^{1} \mathrm{H}$ NMR spectroscopy confirms their structures as the proposed [5+5], [3+3], and [2+2] systems. All of these macrocycles yielded a single, narrow elution band $(Ð<1.05)$ in their GPC traces, consistent with the formation of a single macrocyclic product (Figure 2B). MALDI-MS of each macrocycle showed a single set of peaks corresponding to the expected [5+5], $[3+3]$, or $[2+2]$ macrocycle, with the $[\mathrm{M}+\mathrm{H}]^{+}$adduct being within $0.04 \%$ of the theoretical molecular weight (Figure 2C and Table S1). Each macrocycle was soluble in common organic solvents and exhibited ${ }^{1} \mathrm{H}$ NMR spectra consisting of well-defined resonances with integrations commensurate with the proposed structures (see Supporting Information), along with no resonances corresponding to either free aldehydes or free amines. The ${ }^{1} \mathrm{H}$ NMR, MALDI-MS, and GPC of each macrocycle, as well as their high isolated yields (>90\%), indicate a highly efficient and general macrocyclization process. 


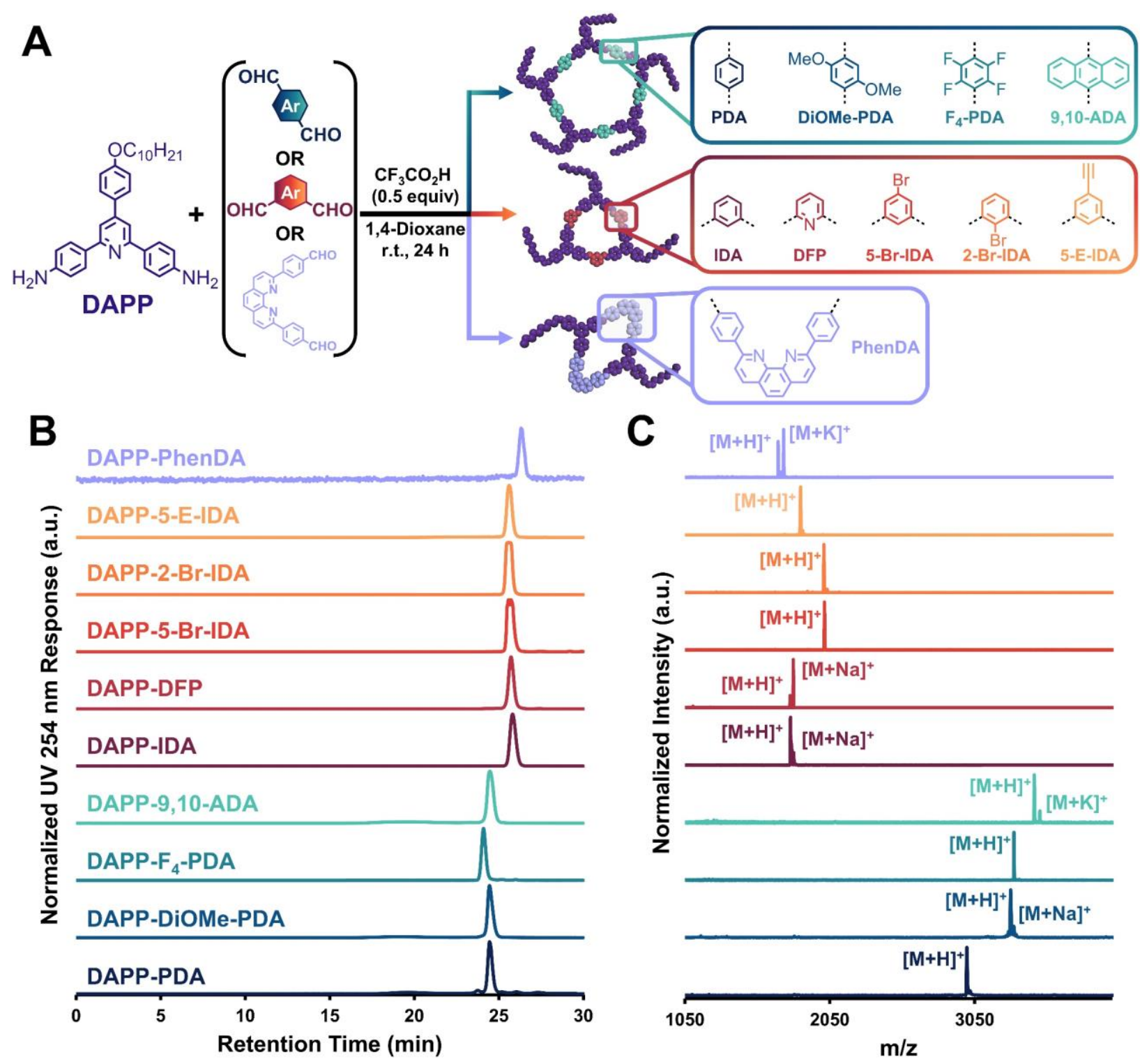

Figure 2. Modular synthesis of imine-linked macrocycles from a pyridine-containing diamine monomer. (A) Scheme of macrocycle synthesis where the shape and size of the macrocycle is dictated by the dialdehyde substitution pattern (B) GPC traces using a $254 \mathrm{~nm}$ ultraviolet (UV) detector. The color of the chromatogram correlates to the color of the dialdehyde monomer. (C) MALDI-MS spectra of the synthesized macrocycles. The color of the spectrum correlates to the color of the dialdehyde monomer.

The use of a pyridine-containing diamine yielded universal access to crystalline nanotubes in a single step, as evaluated by in solvo synchrotron X-ray diffraction (XRD). After allowing the macrocyclization reaction to run for $24 \mathrm{~h}$, the resultant gel was placed in a capillary tube and subjected to XRD measurements. Unassembled macrocycles do not produce observable diffraction. ${ }^{32}$ However, when macrocycles assemble into nanotubes, the emergence of a strong diffraction signal, with a characteristic peak at low values of $q$ related to the macrocycle size (between 0.1 and $\left.0.2 \AA^{-1}\right)^{31-33}$ is observed. Macrocycle assembly was observed following condensation of DAPP with all dialdehydes studied, 
thereby demonstrating that pyridine-based assembly under mildly acidic conditions is general (Figure 3B). The $<100>$ diffraction features of the [3+3] systems, corresponding to the size of the hexagonal pore, were observed at 0.15 to $0.17 \AA^{-1}$, which is consistent with our previous report on similar hexagonal systems. ${ }^{33}$ Due to the increased size of the [5+5] macrocycles, their $<100>$ diffraction feature shifted to lower values of $q\left(0.12\right.$ to $\left.0.13 \AA^{-1}\right)$. Despite having a pentagonal shape, which is classically not able to pack into a periodic array, a diffraction pattern similar to that of the hexagonal systems was observed, which we attribute to the ability of the flexible alkyloxy side chains to fill free volume. Lastly, the primary diffraction feature for the [2+2] PhenDA macrocycle assembly was observed at $0.16 \AA^{-1}$. Despite being comprised of fewer monomers, the shape of PhenDA results in an assembly with an irregularly shaped pore that is similar in size to the hexagonal [3+3] systems. In all cases, the experimentally obtained XRD patterns are in good agreement with a Pawley refined pattern corresponding to macrocycle assembly (see Supporting Information). Overall, the emergence of diffraction signals during the macrocycle synthesis confirms our hypothesis that condensing a pyridine-based diamine with various aromatic dialdehydes enables chemically general tandem macrocyclization and 1D assembly events.

Atomic force microscopy (AFM) and scanning electron microscopy (SEM) confirmed that macrocycles prepared from DAPP and various aromatic dialdehydes assembled into high-aspect ratio nanotubes during their synthesis. While the emergence of XRD signals demonstrated macrocycle assembly, these microscopy techniques show that nanotubes are formed as mesoscale assemblies. Aliquots of all macrocyclizations were drop cast onto silicon wafers and imaged first by AFM, then by SEM (see Supporting Information). In all cases, high-aspect ratio nanotube formation was observed (Figure 3C). ${ }^{32-}$ ${ }^{33}$ From our previous reports, we have shown that the absence of a central pyridine ring prevents longrange assembly under analogous conditions, which suggests that protonation to pyridinium ions drives organization through charge-mediated assembly. ${ }^{33,39}$ These data highlight the modularity of this approach, in that nanotubes with high-aspect ratios were prepared in a single-step despite the constituent macrocycles having different sizes, shapes, and chemical functionalities. 


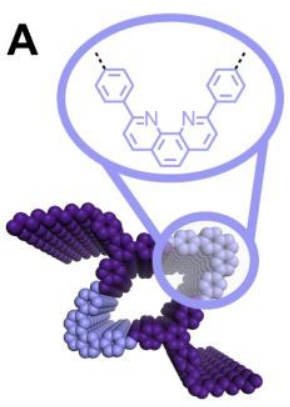

Phenanthrolines [2+2] Macrocycles
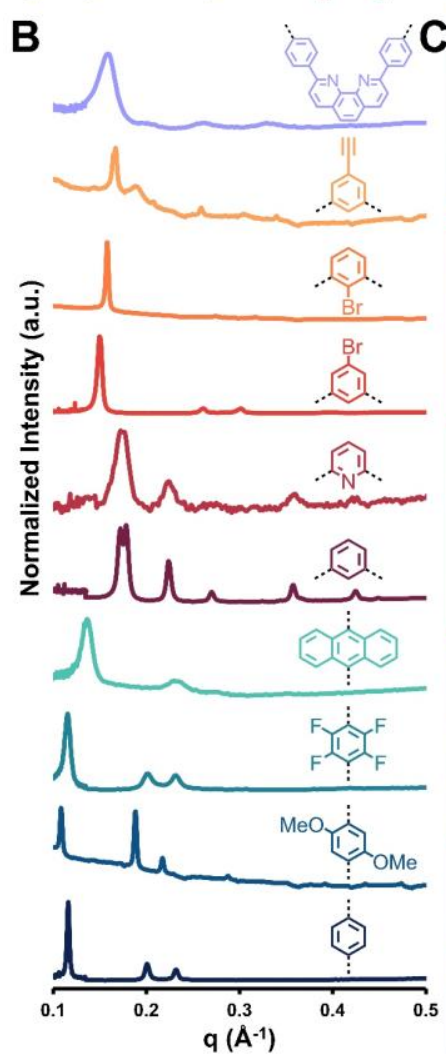

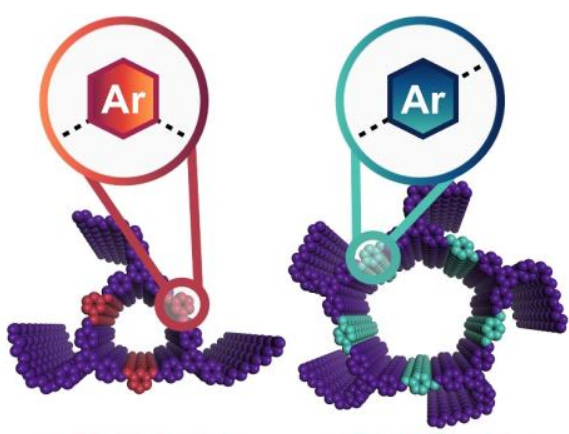

p-Dialdehydes [5+5] Macrocycles

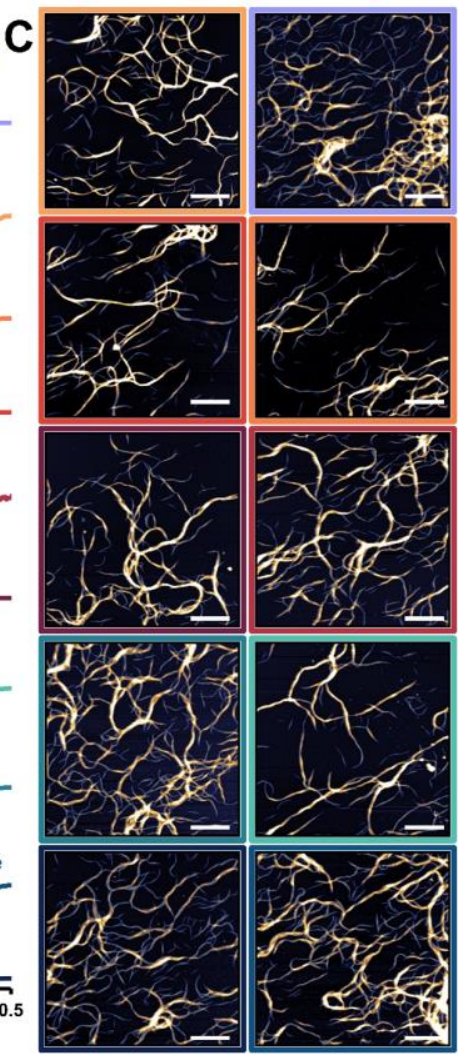

Figure 3. Assembly of imine-linked macrocycles into nanotubes during macrocycle synthesis ( 0.5 equiv $\mathrm{CF}_{3} \mathrm{CO}_{2} \mathrm{H}$ per DAPP). (A) Schematic of nanostructures resulting from the assembly of pentagonal [5+5], hexagonal [3+3], and diamond shaped [2+2] macrocycles. (B) XRD patterns of the synthesized nanotubes accompanied by the structure of the aldehyde monomer. In all cases, the dashed line represents the location of the imine linkage. (C) AFM images of nanotubes with varying dimensions and functionalities. The color of the border correlates to the color of the aldehyde monomer. Scale bars: $7 \mu \mathrm{m}$.

Accessing nanotubes of different sizes and chemical functionalities under sub-stoichiometric acid loadings motivated studies of their proton conductivity. Nanotubes assembled from pyridine-containing iminelinked macrocycles under mildly acidic conditions demonstrated pore size-dependent proton conductivity, with nanotubes assembled from DAPP-IDA exhibiting a conductivity two orders of magnitude greater than nanotubes assembled from DAPP-PDA or DAPP-PhenDA. We performed two-terminal electrochemical impedance spectroscopy (EIS) on various nanotubes at $90 \%$ relative humidity (R.H.) and 
$25^{\circ} \mathrm{C}$, and fit the EIS data to a standard equivalent circuit (Figures $4 \mathrm{~A}$ and $\mathrm{B}$ ). Despite variation in nanotube shape and number of pyridine moieties, nanotubes assembled from DAPP-PDA, DAPP-IDA, and DAPP-PhenDA all yield a semi-circle Nyquist plot in the high-frequency region, and an inclined spur in the low frequency region (Figures 4C-F). These observations are fingerprints of proton conductivity, which is further validated by observing a sharp decrease in conductivity due to isotope effects when the same measurements are carried out in the presence of $\mathrm{D}_{2} \mathrm{O}$ (Figure S134). ${ }^{49-51}$ All samples demonstrate the hallmarks of proton conductivity and our observations suggest that the conductivity values of various nanotubes have a pore size dependence. Larger [5+5] nanotubes $(\varnothing=3 \mathrm{~nm})$ derived from PDA exhibited low conductivity $\left(1.407 \pm 0.030 \times 10^{-5} \mathrm{~S} \mathrm{~m}^{-1}, 90 \%\right.$ R.H., $\left.25^{\circ} \mathrm{C}\right)$. However, the smaller, but irregularly shaped nanotubes $(\varnothing=2.5$ and $1.3 \mathrm{~nm})$ derived from PhenDA only show a moderate conductivity increase $\left(6.279 \pm 0.200 \times 10^{-5} \mathrm{~S} \mathrm{~m}^{-1}, 90 \%\right.$ R.H., $\left.25^{\circ} \mathrm{C}\right)$. The smallest hexagonal nanotubes $(\varnothing=1.75 \mathrm{~nm})$ derived from IDA produced a conductivity two orders of magnitude greater than nanotubes with larger channels $\left(1.598 \pm 0.003 \times 10^{-3} \mathrm{~S} \mathrm{~m}^{-1}, 90 \%\right.$ R.H., $\left.25^{\circ} \mathrm{C}\right)$, suggesting that the size of the hexagonal pore and the spacing of pyridine moieties in nanotubes derived from IDA provide an optimal path for proton conduction. Pore size-dependent proton conductivity within 1D nanochannels has been previously attributed to water molecules adopting a well-organized 1D proton wire configuration in smaller systems, thereby realizing larger conductivity values. ${ }^{52-53}$ Despite the apparent dependence of proton conductivity on nanotube diameter, further studies will be needed to definitively elucidate the origin of enhanced conductivity within various nanotubes.

Comparison of the conductivity trends in nanotubes derived from DAPP with two additional control systems confirmed that proton transport occurs along the cationic 1D nanochannel. Two additional macrocycles were prepared based on a 1,3,5-triphenylbenzene-based diamine (DAPB) and either DFP or IDA. ${ }^{32-33}$ The former is a structural isomer of DAPP-IDA with an identical assembly profile, while the latter lacks pyridine moieties and substantial 1D order, allowing us to further probe the effects of assembly and nanotube size on proton conductivity. Nanotubes assembled from DAPB-DFP macrocycles exhibit substantial proton conductivities $\left(1.488 \pm 0.070 \times 10^{-3} \mathrm{~S} \mathrm{~m}^{-1}, 90 \%\right.$ R.H., $\left.25^{\circ} \mathrm{C}\right)$, similar to DAPP-IDA systems. Furthermore, macrocycles which do not contain any pyridine moieties, and therefore lack substantial 1D order (DAPB-IDA), demonstrate conductivity values at least three order of magnitude lower than DAPP-IDA or DAPB-DFP nanotubes (Figure S135). The comparison of these three hexagonal structures suggests that (1) proton conductivity is partially dependent on the pore architecture and (2) substantial 1D order is required for reliable proton transport. Taken together, the results of the conductivity values obtained for various DAPP-based nanotubes highlight that in order to reliably target optimized 
performance in a 1D nanochannel, the chemical functionality and nanotube topology must be independently modifiable. Presumably, using this design strategy to prepare optimally sized nanotubes with higher densities of basic moieties will improve the proton conductivity of these assemblies, thereby enabling explorations into the use of these materials in fuel cell and bioelectronic applications. ${ }^{54}$ More broadly, this work demonstrates that structurally well-defined supramolecular assemblies with precisely installed chemical functionalities are promising scaffolds for the development of materials suitable for ion transport.
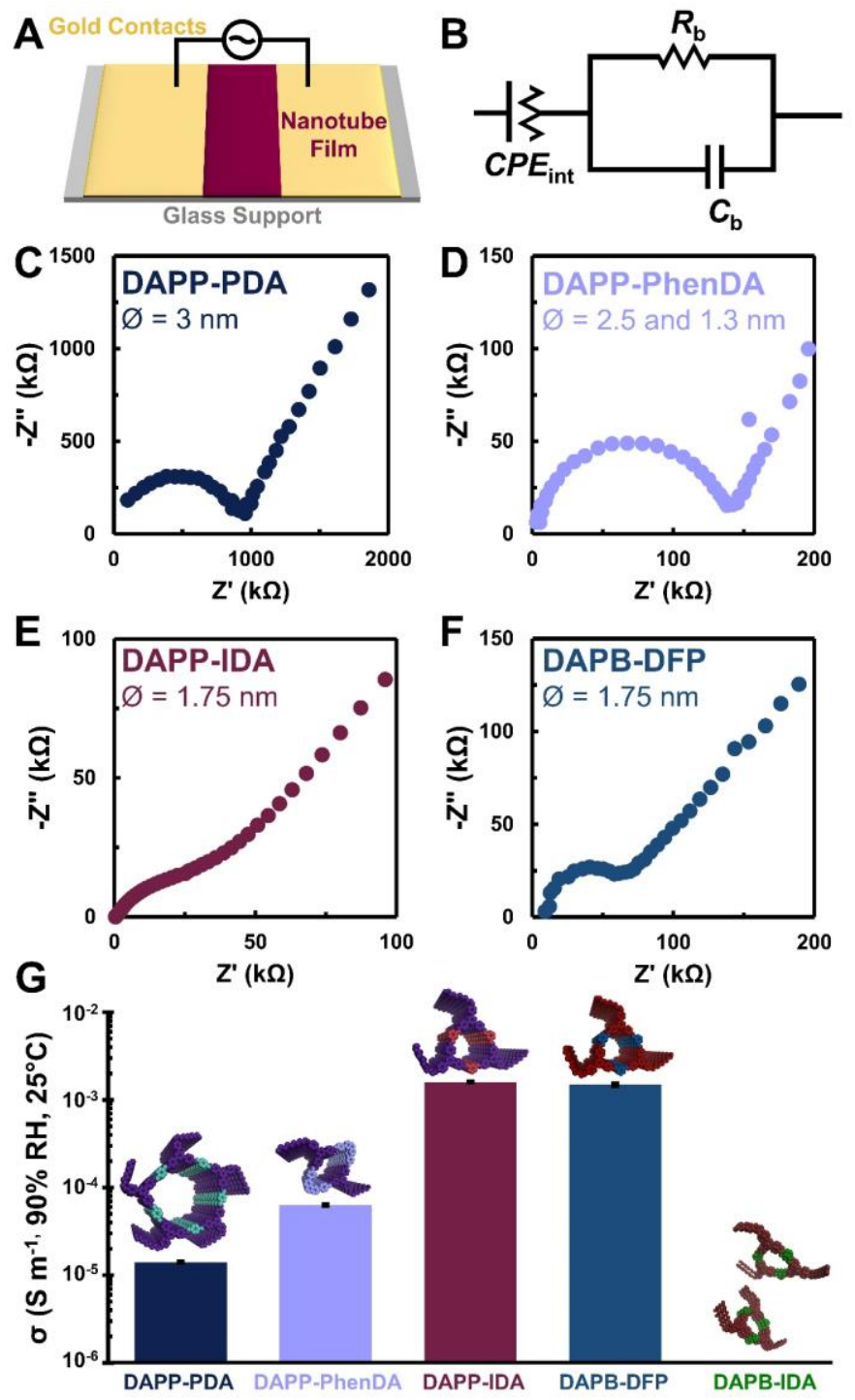

Figure 4. Pore size-dependent proton conductivity of nanotubes prepared via acid-mediated macrocycle assembly. (A) Illustration of the two-terminal device used for EIS measurements. (B) Diagram of the equivalent circuit model used to analyze the impedance data. The circuit consists of a Constant Phase Element $\left(C P E_{\text {int }}\right)$ in series with both a resistor $\left(R_{\mathrm{b}}\right)$ and a capacitor $\left(C_{\mathrm{b}}\right)$, which correspond to the film/electrode interface capacitance, the film bulk resistance, and the film bulk capacitance, respectively. (C-F) Nyquist plots showing the impedance of nanotubes derived from DAPP-PDA, DAPP-PhenDA, DAPP-IDA, and DAPB-DFP macrocycles at $25^{\circ} \mathrm{C}$ with $90 \%$ R.H. The conductivities calculated from 
these plots were $1.407 \times 10^{-5} \mathrm{~S} \mathrm{~m}^{-1}, 6.279 \times 10^{-5} \mathrm{~S} \mathrm{~m}^{-1}, 1.598 \times 10^{-3} \mathrm{~S} \mathrm{~m}^{-1}$, and $1.488 \times 10^{-3} \mathrm{~S} \mathrm{~m}^{-1}$, respectively. (G) Comparison of observed conductivity values highlighting pore size dependence of proton conductivity.

\title{
Conclusions
}

Generalizing the acid-mediated assembly of macrocycles into robust nanotubes will enable a broad range of structures to be accessed and their emergent properties to be explored. Here we have shown that a 2,4,6-triphenylpyridine-based diamine (DAPP) enables efficient formation of pentagonal [5+5], hexagonal [3+3], and diamond shaped [2+2] macrocycles depending on the substitution pattern of the aromatic dialdehyde monomer. Protonation of the central pyridine moiety of DAPP under the mild conditions typical for macrocycle synthesis drives macrocycle assembly into high-aspect ratio nanotubes, as demonstrated by in solvo XRD measurements and AFM imaging. These structurally well-defined supramolecular polymers with cationic 1D nanochannels demonstrated pore size-dependent proton conductivity, with nanotubes derived from IDA exhibiting a conductivity two orders of magnitude greater than nanotubes derived from PDA or PhenDA. These findings, along with the versatility of this synthetic approach, will guide future designs to further improve proton conductivity and further leverage the stimuli-responsive nature of the assemblies. Overall, this platform will unlock the potential of functional nanotubes assembled from macrocyclic precursors to provide systems whose emergent properties and functions can be rationally tuned through molecular design.

\section{ASSOCIATED CONTENT}

Supporting Information. The Supporting Information is available free of charge on the ChemRxiv Server:

Instrumentation, synthetic and experimental procedures, NMR spectra, FTIR spectra, GPC chromatograms, MALDI-MS spectra, in solvo XRD patterns, Pawley structural refinements, SEM images, AFM images, EIS methods and data (PDF)

\author{
AUTHOR INFORMATION \\ Corresponding Authors \\ *MR: mrolandi@ucsc.edu \\ *WRD: wdichtel@northwestern.edu
}




\section{ORCID}

Michael J. Strauss: 0000-0003-0808-2568

Manping Jia: 0000-0001-7299-6220

Austin M. Evans: 0000-0002-3597-2454

Ioannina Castano: 0000-0001-6491-2170

Rebecca L. Li: 0000-0001-7208-3413

Xavier Aguilar-Enriquez: 0000-0002-5940-4427

Emily K. Roesner: 0000-0003-0583-7371

Jeremy L. Swartz: 0000-0003-0391-8434

Alan E. Enciso: 0000-0002-9945-1287

J. Fraser Stoddart: 0000-0003-3161-3697

Marco Rolandi: 0000-0001-7898-2479

William R. Dichtel: 0000-0002-3635-6119

\section{Notes}

The authors declare no competing financial interests.

\section{Acknowledgements}

This work was funded by the Army Research Office through the Multidisciplinary University Research Initiative (MURI; W911NF-15-1-04477, to W.R.D.). M.J.S was supported by the National Science Foundation (NSF) through the Graduate Research Fellowship Program (GRFP) under Grant No. DGE1842165. M.J.S. is partially supported by the Ryan Fellow-ship and the International Institute for Nanotechnology. A.M.E. was supported by the NSF through the GRFP under Grant No. DGE-1324585. I.C. was supported by the NSF through the GRFP under Grant No. DGE-1842165. X.A.E. was supported by the NSF through the GRFP under Grant No. DGE-1842165. J.L.S. was supported by the NSF through the GRFP under Grant No. DGE-1842165. This work made use of the Integrated Molecular Structure Education and Research Center (IMSERC) at Northwestern University, which has re-ceived support from the NSF (CHE-1048773), the Soft and Hybrid Nanotechnology Experimental (SHyNE) Resource (NSF; NNCI-1542205), the State of Illinois, and the Interna-tional Institute for Nanotechnology (IIN). This work also made use of the Scanned Probe Imaging and Development (SPID), and the Electron Probe Instrumentation Center (EPIC), facilities of Northwestern University's Atomic and Nanoscale Characterization Experiment Center (NUANCE), which has received support from the Soft and Hybrid Nano-technology Experimental (SHyNE) Resource (NSF; ECCS-1542205); the MRSEC program (NSF; DMR-1720139) at the Materials Research Center; the International Institute for Nanotechnology (IIN); the Keck Foundation; and the State of Illinois. Instruments used in this work were also supported by the Northwestern University Keck Biophysics Facility and a Cancer Center Support Grant (NCI CA060553). Parts of this work were performed at the DuPont-Northwestern-Dow Col-laborative Access Team (DND- 
CAT) located at Sector 5 of the Advanced Photon Source (APS) at Argonne National Lab. This research used resources of the Advanced Photon Source and the Center for Nanoscale Materials, both U.S. Department of Energy (DOE) Office of Science User Facilities operat-ed for the DOE Office of Science by Argonne National Labora-tory under Grant No. DGE-1324585. M.J. and M.R. thank the W.M. Keck Center for Nanoscale Optofluidics, the California Institute for Quantitative Biosciences (QB3), and the Army Research Office under award number W911NF-17-1-0460 for equipment and facilities support, and funding from the Defense Advanced Research Projects Agency (DARPA) and the Department of Interior under award No. D20AC00003. We acknowledge Prof. Julia Kalow for the use of her GPC in-strument. We also acknowledge Prof. Christine Luscombe for helpful discussions.

\section{References}

1. $\quad$ Iijima, S., Helical microtubules of graphitic carbon. Nature 1991, 354, 56-58.

2. Sato, K.; Itoh, Y.; Aida, T., Columnarly Assembled Liquid-Crystalline Peptidic Macrocycles Unidirectionally Orientable over a Large Area by an Electric Field. J. Am. Chem. Soc. 2011, 133, 1376713769.

3. Fischer, M.; Lieser, G.; Rapp, A.; Schnell, I.; Mamdouh, W.; De Feyter, S.; De Schryver, F. C.; Höger, S., Shape-Persistent Macrocycles with Intraannular Polar Groups: Synthesis, Liquid Crystallinity, and 2D Organization. J. Am. Chem. Soc. 2004, 126, 214-222.

4. Stępień, M.; Donnio, B.; Sessler, J. L., Supramolecular Liquid Crystals Based on Cyclo[8]pyrrole. Angew. Chem. Int. Ed. 2007, 46, 1431-1435.

5. Zhang, J.; Moore, J. S., Nanoarchitectures. 6. Liquid Crystals Based on Shape-Persistent Macrocyclic Mesogens. J. Am. Chem. Soc. 1994, 116, 2655-2656.

6. Shimizu, T.; Ding, W.; Kameta, N., Soft-Matter Nanotubes: A Platform for Diverse Functions and Applications. Chem. Rev. 2020, 120, 2347-2407.

7. Helsel, A. J.; Brown, A. L.; Yamato, K.; Feng, W.; Yuan, L.; Clements, A. J.; Harding, S. V.; Szabo, G.; Shao, Z.; Gong, B., Highly Conducting Transmembrane Pores Formed by Aromatic Oligoamide Macrocycles. J. Am. Chem. Soc. 2008, 130, 15784-15785.

8. Xu, H.; Nagasaka, S.; Kameta, N.; Masuda, M.; Ito, T.; Higgins, D. A., Imaging fluorescence correlation spectroscopy studies of dye diffusion in self-assembled organic nanotubes. Phys. Chem. Chem. Phys. 2016, 18, 16766-16774.

9. Xu, H.; Nagasaka, S.; Kameta, N.; Masuda, M.; Ito, T.; Higgins, D. A., Spectroscopic imaging studies of nanoscale polarity and mass transport phenomena in self-assembled organic nanotubes. Phys. Chem. Chem. Phys. 2017, 19, 20040-20048.

10. de la Rica, R.; Mendoza, E.; Matsui, H., Bioinspired Target-Specific Crystallization on Peptide Nanotubes for Ultrasensitive Pb Ion Detection. Small 2010, 6, 1753-1756.

11. Kameta, N.; Masuda, M.; Shimizu, T., Two-step naked-eye detection of lectin by hierarchical organization of soft nanotubes into liquid crystal and gel phases. Chem. Commun. 2015, 51, 6816-6819.

12. Hu, Y.; Ma, X.; Zhang, Y.; Che, Y.; Zhao, J., Detection of Amines with Fluorescent Nanotubes: Applications in the Assessment of Meat Spoilage. ACS Sens. 2016, 1, 22-25.

13. Yang, J.; Dewal, M. B.; Shimizu, L. S., Self-Assembling Bisurea Macrocycles Used as an Organic Zeolite for a Highly Stereoselective Photodimerization of 2-Cyclohexenone. J. Am. Chem. Soc. 2006, 128, 8122-8123. 
14. Xu, Y.; Wang, T.; He, Z.; Zhong, A.; Yu, W.; Shi, B.; Huang, K., Synthesis of triphenylphosphinebased microporous organic nanotube framework supported Pd catalysts with excellent catalytic activity. Polym. Chem. 2016, 7, 7408-7415.

15. Kameta, N.; Dong, J.; Yui, H., Thermoresponsive PEG-Coated Nanotubes as Chiral Selectors of Amino Acids and Peptides. Small 2018, 14, 1800030.

16. Kameta, N.; Ding, W.; Dong, J., Soft Nanotubes Derivatized with Short PEG Chains for Thermally Controllable Extraction and Separation of Peptides. ACS Omega 2017, 2, 6143-6150.

17. Leonhardt, E. J.; Van Raden, J. M.; Miller, D.; Zakharov, L. N.; Alemán, B.; Jasti, R., A BottomUp Approach to Solution-Processed, Atomically Precise Graphitic Cylinders on Graphite. Nano Lett. 2018, 18, 7991-7997.

18. Ghadiri, M. R.; Granja, J. R.; Milligan, R. A.; McRee, D. E.; Khazanovich, N., Self-assembling organic nanotubes based on a cyclic peptide architecture. Nature 1993, 366, 324-327.

19. Lahiri, S.; Thompson, J. L.; Moore, J. S., Solvophobically Driven $\pi$-Stacking of Phenylene Ethynylene Macrocycles and Oligomers. J. Am. Chem. Soc. 2000, 122, 11315-11319.

20. Shimizu, L. S.; Hughes, A. D.; Smith, M. D.; Davis, M. J.; Zhang, B. P.; zur Loye, H.-C.; Shimizu, K. D., Self-Assembled Nanotubes that Reversibly Bind Acetic Acid Guests. J. Am. Chem. Soc. 2003, 125, 14972-14973.

21. Gallant, A. J.; MacLachlan, M. J., Ion-Induced Tubular Assembly of Conjugated Schiff-Base Macrocycles. Angew. Chem. Int. Ed. 2003, 42, 5307-5310.

22. Rubin, D. J.; Amini, S.; Zhou, F.; Su, H.; Miserez, A.; Joshi, N. S., Structural, Nanomechanical, and Computational Characterization of D,L-Cyclic Peptide Assemblies. ACS Nano 2015, 9, 3360-3368.

23. Balkenende, D. W. R.; Monnier, C. A.; Fiore, G. L.; Weder, C., Optically responsive supramolecular polymer glasses. Nat. Commun. 2016, 7, 10995.

24. Albertazzi, L.; van der Veeken, N.; Baker, M. B.; Palmans, A. R. A.; Meijer, E. W., Supramolecular copolymers with stimuli-responsive sequence control. Chem. Commun. 2015, 51 , 1616616168.

25. Webber, M. J., Engineering responsive supramolecular biomaterials: Toward smart therapeutics. Bioeng. Transl. Med. 2016, 1, 252-266.

26. Kumar, M.; Brocorens, P.; Tonnelé, C.; Beljonne, D.; Surin, M.; George, S. J., A dynamic supramolecular polymer with stimuli-responsive handedness for in situ probing of enzymatic ATP hydrolysis. Nat. Commun. 2014, 5, 5793.

27. Wanzke, C.; Jussupow, A.; Kohler, F.; Dietz, H.; Kaila, V. R. I.; Boekhoven, J., Dynamic Vesicles Formed by Dissipative Self-Assembly. ChemSystemsChem 2020, 2, e1900044.

28. Dai, K.; Fores, J. R.; Wanzke, C.; Winkeljann, B.; Bergmann, A. M.; Lieleg, O.; Boekhoven, J., Regulating Chemically Fueled Peptide Assemblies by Molecular Design. J. Am. Chem. Soc. 2020, 142, 14142-14149.

29. Kriebisch, B. A. K.; Jussupow, A.; Bergmann, A. M.; Kohler, F.; Dietz, H.; Kaila, V. R. I.; Boekhoven, J., Reciprocal Coupling in Chemically Fueled Assembly: A Reaction Cycle Regulates SelfAssembly and Vice Versa. J. Am. Chem. Soc. 2020, 142, 20837-20844.

30. van Rossum, S. A. P.; Tena-Solsona, M.; van Esch, J. H.; Eelkema, R.; Boekhoven, J., Dissipative out-of-equilibrium assembly of man-made supramolecular materials. Chem. Soc. Rev. 2017, 46, 55195535.

31. Sun, C.; Shen, M.; Chavez, A. D.; Evans, A. M.; Liu, X.; Harutyunyan, B.; Flanders, N. C.; Hersam, M. C.; Bedzyk, M. J.; Olvera de la Cruz, M.; Dichtel, W. R., High aspect ratio nanotubes assembled from macrocyclic iminium salts. Proc. Natl. Acad. Sci. U.S.A. 2018, 115, 8883-8888.

32. Strauss, M. J.; Asheghali, D.; Evans, A. M.; Li, R. L.; Chavez, A. D.; Sun, C.; Becker, M. L.; Dichtel, W. R., Cooperative Self-Assembly of Pyridine-2,6-Diimine-Linked Macrocycles into Mechanically Robust Nanotubes. Angew. Chem. Int. Ed. 2019, 58, 14708-14714. 
33. Strauss, M. J.; Evans, A. M.; Castano, I.; Li, R. L.; Dichtel, W. R., Supramolecular polymerization provides non-equilibrium product distributions of imine-linked macrocycles. Chem. Sci. 2020, 11, 19571963.

34. Merí-Bofí, L.; Royuela, S.; Zamora, F.; Ruiz-González, M. L.; Segura, J. L.; Muñoz-Olivas, R.; Mancheño, M. J., Thiol grafted imine-based covalent organic frameworks for water remediation through selective removal of $\mathrm{Hg}(\mathrm{II})$. J. Mat. Chem. A. 2017, 5, 17973-17981.

35. Huang, N.; Krishna, R.; Jiang, D., Tailor-Made Pore Surface Engineering in Covalent Organic Frameworks: Systematic Functionalization for Performance Screening. J. Am. Chem. Soc. 2015, 137, 7079-7082.

36. Lowe, A. B., Thiol-yne 'click'/coupling chemistry and recent applications in polymer and materials synthesis and modification. Polymer 2014, 55, 5517-5549.

37. Lowe, A. B.; Hoyle, C. E.; Bowman, C. N., Thiol-yne click chemistry: A powerful and versatile methodology for materials synthesis. J. Mat. Chem. 2010, 20, 4745-4750.

38. Wang, Y.; Zhang, S.; Wang, L.; Zhang, W.; Zhou, N.; Zhang, Z.; Zhu, X., The Suzuki Coupling Reaction as a Post-Polymerization Modification: A Promising Protocol for Construction of Cyclic-Brush and More Complex Polymers. Polym. Chem. 2015, 6, 4669-4677.

39. Chavez, A. D.; Evans, A. M.; Flanders, N. C.; Bisbey, R. P.; Vitaku, E.; Chen, L. X.; Dichtel, W. R., Equilibration of Imine-Linked Polymers to Hexagonal Macrocycles Driven by Self-Assembly. Chem. Eur. J. 2018, 24, 3989-3993.

40. Schiavo, E.; Muñoz-García, A. B.; Barone, V.; Vittadini, A.; Casarin, M.; Forrer, D.; Pavone, M., Tuning dispersion correction in DFT-D2 for metal-molecule interactions: A tailored reparameterization strategy for the adsorption of aromatic systems on $\operatorname{Ag}\left(\begin{array}{lll}1 & 1 & 1\end{array}\right)$. Chem. Phys. Lett. 2018, 693, 28-33.

41. Weck, M.; Dunn, A. R.; Matsumoto, K.; Coates, G. W.; Lobkovsky, E. B.; Grubbs, R. H., Influence of Perfluoroarene-Arene Interactions on the Phase Behavior of Liquid Crystalline and Polymeric Materials. Angew. Chem. Int. Ed. 1999, 38, 2741-2745.

42. Elacqua, E.; Geberth, G. T.; Vanden Bout, D. A.; Weck, M., Synthesis and Folding Behaviour of Poly(p-phenylene vinylene)-Based $\beta$-sheet Polychromophores. Chem. Sci. 2019, 10, 2144-2152.

43. Wang, S.; Chavez, A. D.; Thomas, S.; Li, H.; Flanders, N. C.; Sun, C.; Strauss, M. J.; Chen, L. X.; Markvoort, A. J.; Bredas, J.-L.; Dichtel, W. R., Pathway Complexity in the Stacking of Imine-Linked Macrocycles Related to Two-Dimensional Covalent Organic Frameworks. Chem. Mater. 2019, 31, 71047111.

44. Xu, H.; Gao, J.; Jiang, D., Stable, crystalline, porous, covalent organic frameworks as a platform for chiral organocatalysts. Nat. Chem. 2015, 7, 905-912.

45. Nagaoka, M.; Tsurumaki, E.; Nishiuchi, M.; Iwanaga, T.; Toyota, S., Synthesis and Electronic Properties of Length-Defined 9,10-Anthrylene-Butadiynylene Oligomers. J. Org. Chem. 2018, 83, 57845790.

46. Haldar, S.; Chakraborty, D.; Roy, B.; Banappanavar, G.; Rinku, K.; Mullangi, D.; Hazra, P.; Kabra, D.; Vaidhyanathan, R., Anthracene-Resorcinol Derived Covalent Organic Framework as Flexible White Light Emitter. J. Am. Chem. Soc. 2018, 140, 13367-13374.

47. Meyer, M.; Albrecht-Gary, A.-M.; Dietrich-Buchecker, C. O.; Sauvage, J.-P., $\pi-\pi$ StackingInduced Cooperativity in Copper(I) Complexes with Phenanthroline Ligands. Inorg. Chem. 1999, 38, 2279-2287.

48. Liu, Y.; Ma, Y.; Zhao, Y.; Sun, X.; Gándara, F.; Furukawa, H.; Liu, Z.; Zhu, H.; Zhu, C.; Suenaga, K.; Oleynikov, P.; Alshammari, A. S.; Zhang, X.; Terasaki, O.; Yaghi, O. M., Weaving of organic threads into a crystalline covalent organic framework. Science 2016, 351, 365-369.

49. $\quad$ Dusastre, V., Materials for Sustainable Energy. Nature Publishing Group, U.K. 2011.

50. Selberg, J.; Jia, M.; Rolandi, M., Proton conductivity of glycosaminoglycans. PLOS ONE 2019, 14, e0202713. 
51. Phillips, M.; Wheeler, A.; Robinson, M. J.; Leppert, V.; Jia, M.; Rolandi, M.; Hirst, L. S.; Amemiya, C. T., Structural Characteristics and Proton Conductivity of the Gel Within the Electrosensory Organs of Cartilaginous Fishes. bioRxiv 2021, 2021.01.04.425255.

52. Miyake, T.; Rolandi, M., Grotthuss mechanisms: from proton transport in proton wires to bioprotonic devices. J. Phys. Condens. Matter 2015, 28, 023001.

53. Zhong, C.; Deng, Y.; Roudsari, A. F.; Kapetanovic, A.; Anantram, M. P.; Rolandi, M., A polysaccharide bioprotonic field-effect transistor. Nat. Commun. 2011, 2, 476.

54. Jia, M.; Rolandi, M., Soft and Ion-Conducting Materials in Bioelectronics: From Conducting Polymers to Hydrogels. Adv. Healthcare Mater. 2020, 9, 1901372. 